\title{
The dependence of cast structure on geometric shape, vibration conditions and thermophysical properties of mold
}

\author{
L J IVANIĆ, B KOČOVSKI and Z KILIBARDA \\ Technical faculty in Bor, University of Belgrade, V. J. 12, YU-19210 Bor, Yugoslavia \\ MS received 7 April 1995
}

\begin{abstract}
Ibstract. This paper contains results of experiments carried out with the aim of assessing the influence of various casting parameters on the microstructure of an aluminium alloy. Castings have been made with and without vibration, in molds with different thermophysical properties.
\end{abstract}

Keywords. Structure; vibration; type of cast.

\section{Introduction}

It is well known that the primary alm during the crystallization process of aluminium alloy containing $4.5 \%$ copper is to achieve a fine grain structure. To this end, the influence of vibration parameters (amplitude and frequency) as well as of the geometry of the mold on the grain size have earlier been considered (Gončarevic 1986). The objective of the present study is to estimate the influence of thermophysical features, vibration parameters and mold shape on the resultant grain size in $\mathrm{Al}-4.5 \mathrm{Cu}$ alloy.

\section{Experimental}

The structure of $\mathrm{Al}-4.5 \mathrm{Cu}$ alloy consists of $\alpha$-solid solution and grain boundary intermetallic compound $\mathrm{CuAl}_{2}$ ( $\delta$-phase) which enables easy counting of the grains. Casting was done in sand, graphite or metal $(\mathrm{Al}-4.5 \mathrm{Cu})$ mold. Stationary as well as vibration conditions were employed during solidification. The cooling modulus of different geometric shaped molds (roller, cube, slab or ball) was maintained constant with a view to obtain the same heat-technical conditions for the solidification process of the metal bath. The three-factor experiment plan (Krasovskij 1982) presented in figure 1 is followed. Fixing the value of the modulus of cooling $\left(M_{\mathrm{cos}}=1\right)$ for cubic (side of length $60 \mathrm{~mm}$ ) mold, the dimensions of other mold shapes were estimated (Ivanić and Kočovski 1993). Maintaining a constant value for $M_{\text {cool }}$, i.e. equality of ratio between the volume and area of cooling of castings, is desirable in order to avoid the influence of mass and heat variations and ensure approximately equal heat-technical conditions (Krivandina 1986).

The three-factor plan of experiments includes the variations of the following parameters:
A. Vibration parameters
- Amplitude (factor $A$ ); $0.2,0.8$ and $1.2 \mathrm{~mm}$
- Frequency (factor $B$ ); 8,16 and $24 \mathrm{~Hz}$
B. Thermophysical mold properties (factor $C$ )
- Casting in sand-clay molds for which the value of accumulation heat coefficient $b_{2}=100 \mathrm{~J} / \mathrm{m}^{2} \mathrm{~s}^{1 / 2} \mathrm{~K}$.



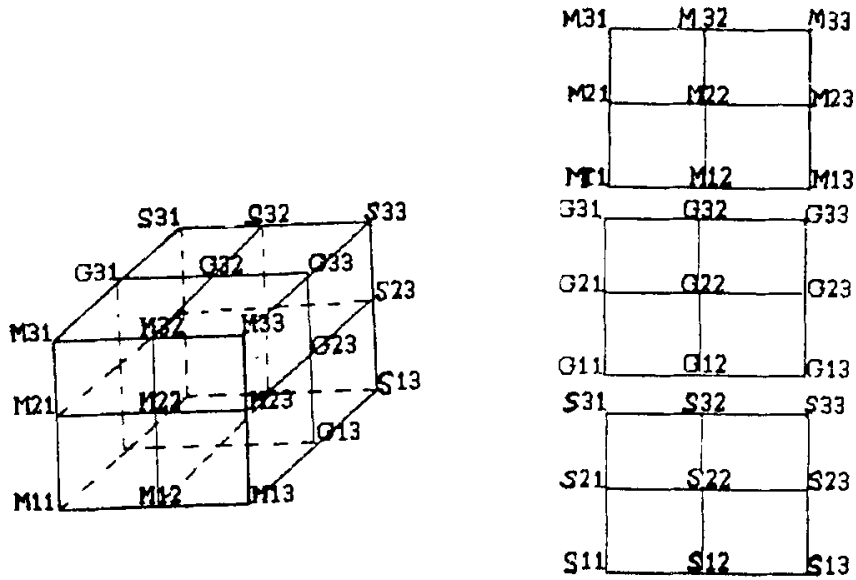

Figure 1. Schematic presentation of three-factor experimental plan.

Table 1. The dependence of number of grains $\left(\mathrm{mm}^{-2}\right)$ on geometric shape of casting, vibration parameters and type of mold.

\begin{tabular}{|c|c|c|c|c|c|c|c|c|c|}
\hline \multirow{3}{*}{$\begin{array}{l}\text { Geometric } \\
\text { shape } \\
\text { of casting } \\
\text { Factor C } \\
\text { Type of mold }\end{array}$} & \multicolumn{9}{|c|}{ Factor $\Lambda$-amplitude $A, \mathrm{~mm}$} \\
\hline & \multicolumn{3}{|c|}{$\begin{array}{c}0 \cdot 2 \\
\text { Factor B } \\
\text { Frequency, } \mathrm{Hz}\end{array}$} & \multicolumn{3}{|c|}{$\begin{array}{c}0.8 \\
\text { Factor B } \\
\text { Frequency, Hz }\end{array}$} & \multicolumn{3}{|c|}{$\begin{array}{c}1 \cdot 4 \\
\text { Factor B } \\
\text { Frequency, Hz. }\end{array}$} \\
\hline & 8 & 16 & 24 & 8 & 16 & 24 & 8 & 16 & 24 \\
\hline Cube: & 294 & 325 & 316 & 308 & 332 & 347 & 333 & 350 & 359 \\
\hline Metallic & 297 & 326 & 321 & 314 & 335 & 347 & 340 & 350 & 366 \\
\hline Graphite & $\begin{array}{l}165 \\
160\end{array}$ & $\begin{array}{l}180 \\
175\end{array}$ & $\begin{array}{l}190 \\
188\end{array}$ & $\begin{array}{l}178 \\
181\end{array}$ & $\begin{array}{l}198 \\
201\end{array}$ & $\begin{array}{l}210 \\
221\end{array}$ & $\begin{array}{l}209 \\
206\end{array}$ & $\begin{array}{l}220 \\
223\end{array}$ & $\begin{array}{l}252 \\
246\end{array}$ \\
\hline Sand & $\begin{array}{l}33 \\
27\end{array}$ & $\begin{array}{l}52 \\
45\end{array}$ & $\begin{array}{l}66 \\
62\end{array}$ & $\begin{array}{l}46 \\
46\end{array}$ & $\begin{array}{l}61 \\
59\end{array}$ & $\begin{array}{l}85 \\
80\end{array}$ & $\begin{array}{l}55 \\
52\end{array}$ & $\begin{array}{l}83 \\
78\end{array}$ & $\begin{array}{l}130 \\
125\end{array}$ \\
\hline $\begin{array}{l}\text { Roller: } \\
\text { Metallic }\end{array}$ & $\begin{array}{l}243 \\
236\end{array}$ & $\begin{array}{l}224 \\
239\end{array}$ & $\begin{array}{l}265 \\
262\end{array}$ & $\begin{array}{l}248 \\
248\end{array}$ & $\begin{array}{l}353 \\
256\end{array}$ & $\begin{array}{l}268 \\
272\end{array}$ & $\begin{array}{l}253 \\
260\end{array}$ & $\begin{array}{l}267 \\
274\end{array}$ & $\begin{array}{l}291 \\
294\end{array}$ \\
\hline Graphite & $\begin{array}{l}124 \\
129\end{array}$ & $\begin{array}{l}140 \\
142\end{array}$ & $\begin{array}{l}150 \\
148\end{array}$ & $\begin{array}{l}130 \\
132\end{array}$ & $\begin{array}{l}148 \\
150\end{array}$ & $\begin{array}{l}160 \\
163\end{array}$ & $\begin{array}{l}159 \\
164\end{array}$ & $\begin{array}{l}176 \\
169\end{array}$ & $\begin{array}{l}190 \\
196\end{array}$ \\
\hline Sand & $\begin{array}{l}16 \\
18\end{array}$ & $\begin{array}{l}25 \\
24\end{array}$ & $\begin{array}{l}40 \\
38\end{array}$ & $\begin{array}{l}24 \\
27\end{array}$ & $\begin{array}{l}34 \\
36\end{array}$ & $\begin{array}{l}45 \\
47\end{array}$ & $\begin{array}{l}33 \\
34\end{array}$ & $\begin{array}{l}43 \\
46\end{array}$ & $\begin{array}{l}67 \\
65\end{array}$ \\
\hline $\begin{array}{l}\text { Slab: } \\
\text { Metallic }\end{array}$ & $\begin{array}{l}325 \\
323\end{array}$ & $\begin{array}{l}333 \\
342\end{array}$ & $\begin{array}{l}350 \\
366\end{array}$ & $\begin{array}{l}338 \\
338\end{array}$ & $\begin{array}{l}344 \\
356\end{array}$ & $\begin{array}{l}376 \\
379\end{array}$ & $\begin{array}{l}340 \\
344\end{array}$ & $\begin{array}{l}352 \\
362\end{array}$ & $\begin{array}{l}383 \\
393\end{array}$ \\
\hline Graphite & $\begin{array}{l}213 \\
210\end{array}$ & $\begin{array}{l}218 \\
222\end{array}$ & $\begin{array}{l}235 \\
230\end{array}$ & $\begin{array}{l}226 \\
230\end{array}$ & $\begin{array}{l}293 \\
240\end{array}$ & $\begin{array}{l}280 \\
276\end{array}$ & $\begin{array}{l}252 \\
248\end{array}$ & $\begin{array}{l}280 \\
265\end{array}$ & $\begin{array}{l}328 \\
322\end{array}$ \\
\hline Sand & $\begin{array}{l}30 \\
33\end{array}$ & $\begin{array}{l}58 \\
54\end{array}$ & $\begin{array}{l}94 \\
90\end{array}$ & $\begin{array}{l}39 \\
43\end{array}$ & $\begin{array}{l}70 \\
71\end{array}$ & $\begin{array}{l}134 \\
103\end{array}$ & $\begin{array}{l}60 \\
57\end{array}$ & $\begin{array}{l}82 \\
82\end{array}$ & $\begin{array}{l}142 \\
139\end{array}$ \\
\hline $\begin{array}{l}\text { Ball: } \\
\text { Metallic }\end{array}$ & $\begin{array}{l}436 \\
444\end{array}$ & $\begin{array}{l}496 \\
497\end{array}$ & $\begin{array}{l}447 \\
454\end{array}$ & $\begin{array}{l}462 \\
467\end{array}$ & $\begin{array}{l}519 \\
513\end{array}$ & $\begin{array}{l}573 \\
576\end{array}$ & $\begin{array}{l}475 \\
479\end{array}$ & $\begin{array}{l}556 \\
556\end{array}$ & $\begin{array}{l}615 \\
619\end{array}$ \\
\hline Graphite & $\begin{array}{l}249 \\
245\end{array}$ & $\begin{array}{l}296 \\
284\end{array}$ & $\begin{array}{l}328 \\
320\end{array}$ & $\begin{array}{l}296 \\
290\end{array}$ & $\begin{array}{l}338 \\
342\end{array}$ & $\begin{array}{l}356 \\
360\end{array}$ & $\begin{array}{l}346 \\
339\end{array}$ & $\begin{array}{l}360 \\
353\end{array}$ & $\begin{array}{l}403 \\
410\end{array}$ \\
\hline Sand & $\begin{array}{l}42 \\
41\end{array}$ & $\begin{array}{l}82 \\
82\end{array}$ & $\begin{array}{l}102 \\
105\end{array}$ & $\begin{array}{l}49 \\
50\end{array}$ & $\begin{array}{l}84 \\
80\end{array}$ & $\begin{array}{l}130 \\
127\end{array}$ & $\begin{array}{l}61 \\
63\end{array}$ & $\begin{array}{l}105 \\
109\end{array}$ & $\begin{array}{l}154 \\
149\end{array}$ \\
\hline
\end{tabular}



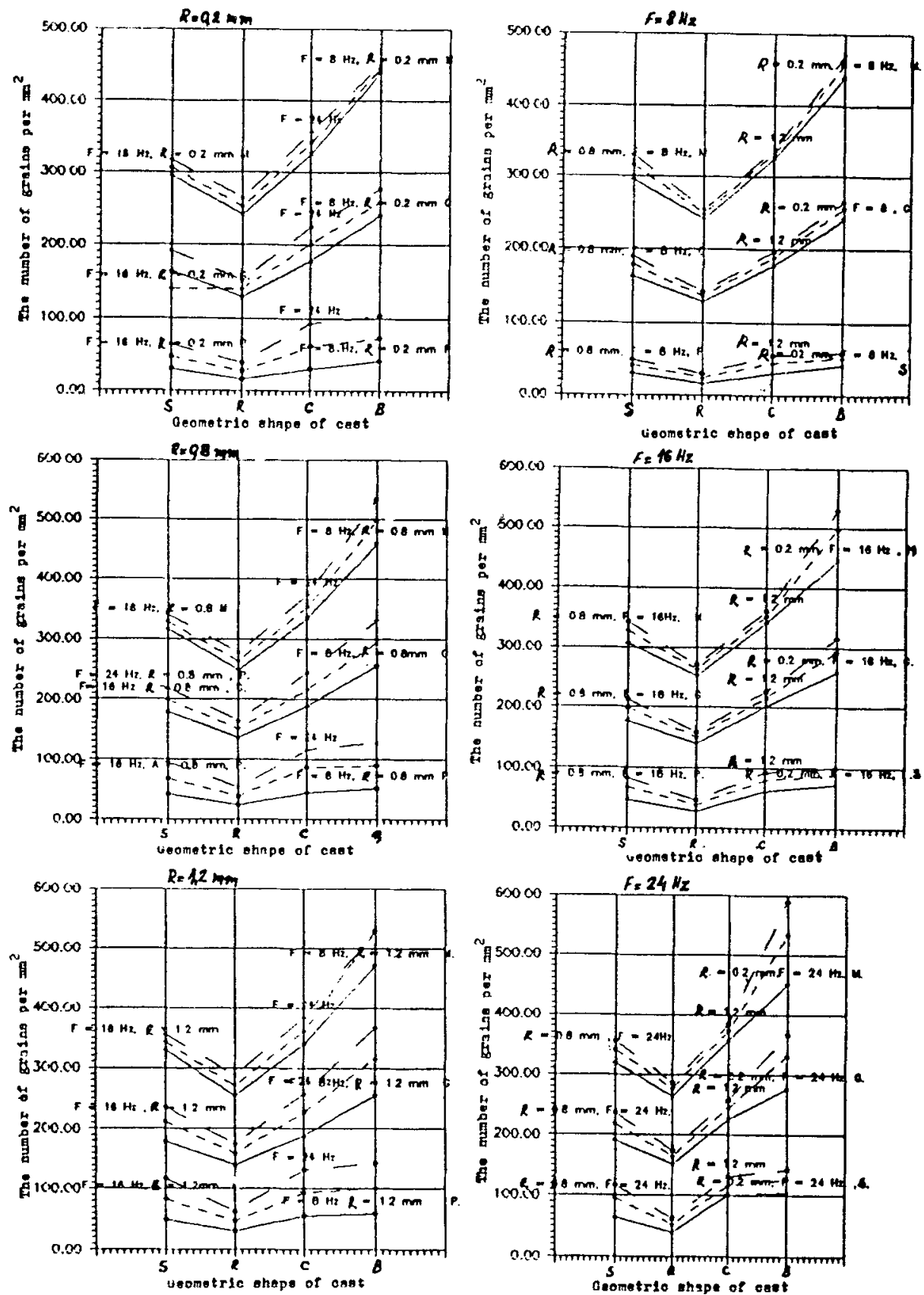

Figure 2. Variation of grain size with geometric shape of casting, vibration parameters and type of mold.

Casting in different shapes of graphite molds, $b_{2}=185 \mathrm{~J} / \mathrm{m}^{2} \mathrm{~s}^{1 / 2} \mathrm{~K}$.

Casting in different shapes of metallic molds, $b_{2}=364 \mathrm{~J} / \mathrm{m}^{2} \mathrm{~s}^{1 / 2} \mathrm{~K}$. 
C. Geometric shapes of the casts - cube, slab, roller and ball. Each experiment was repeated three times. The cast structure in each case was examined under optical microscope in order to estimate the number of grains per unit area.

\section{Results and discussion}

The experimental results gathered have been subjected to a regression analysis by a computer. These data on the effect of various parameters on the cast structure are presented in table 1 in terms of the number of grains per unit area. The values reveal that there exists a strong dependence of the grain size on the mold shape, vibration parameters and thermophysical properties of the mold. Figure 2 brings out more clearly this influence. Conditions favourable for minimizing the grain size should therefore be selected in practice. In general, metallic molds and slab or ball shape for the castings in conjunction with high frequency vibration are conducive for the production of fine grained castings.

The influence of vibration on the structure of castings has been explained in terms of the spreading of waves and reflection from the cast surfaces which increase the number of crystallization centres. Similarly the mold shape and material affect the heat transfer characteristics, which in turn determine the density of crystallization nuclei.

\section{References}

Gončarević I F 1986 Nestandardni put. Moscow'

Ivanić Lj and Kočovski B 1993 Studija uticaja različitih faktora na strukturu formiranu pri kristalizaciji rastopa sa $4.5 \%$ bakra, Bor

Krasovskij G I 1982 Planirovanic eksperimenta, Minsk

Krivandina V A 1986 Metalurška toplotna tehnika, Moscow 